\title{
Do the high costs of implants influence patients' decisions? A cross-sectional study in Saudi Arabia
}

\section{Alzahrani, Abdullah Ali H}

BSc(DT), MDPH, PhD, Dental Health Department, School of Applied Medical Sciences, Al Baha University, Saudi Arabia.

\section{Background and Aim}

\section{Background}

- The decision-making process surrounding a patient's suitability for dental implant is complex.

- Evidence-based dentistry considers patient's social and psychological aspects, and best existing evidence to guide the final decision.

- Aspects such as the patient's oral hygiene, appearance and demographic details were associated with the patient's decision to undertake implant therapy.

- No studies in the literature examining how high costs of implant care influences patients' decisions, particularly when other inexpensive alternatives, such as removable dentures and fixed partial dentures, are possible.

\section{Aim}

To explores how the high costs of dental implants in Saudi Arabia influence patients' decisions.

\section{Objectives}

1) To explore factors associated with patients' decisions to have implants

2) To evaluate association between high costs of implants and the decisions to have the implant care.

\section{Methods and Materials}

- Across sectional study that adopted a qualitative method using semi-structured interviews.

- A purposive sample of Saudi patients who were interested in implant care was used.

- The study involved purposive sampling of a wide range of consultations, including males and females.

- Interviews were audiotaped and transcribed.

- Data were analyzed using inductive thematic analysis and the framework method.

\section{Results}

- Forty-two patients and five dentists contributed to this study.

- There were differences in how patients thought about the implant decisions.

- The high costs of implant care influenced the patients' decisions to undertake this therapy.

- Patients showed great interest in dental implants; however, not all could have them due to social, psychological and, most commonly, financial factors associated with implant care (see figure 1).
- Social and psychological factors included fear of implant failure, fear of the surgical part of this therapy and patient anxiety.

- The majority of the patients, and particularly those from lower socioeconomic status, indicated interest in implants, they were unable to afford them (see figure 2).

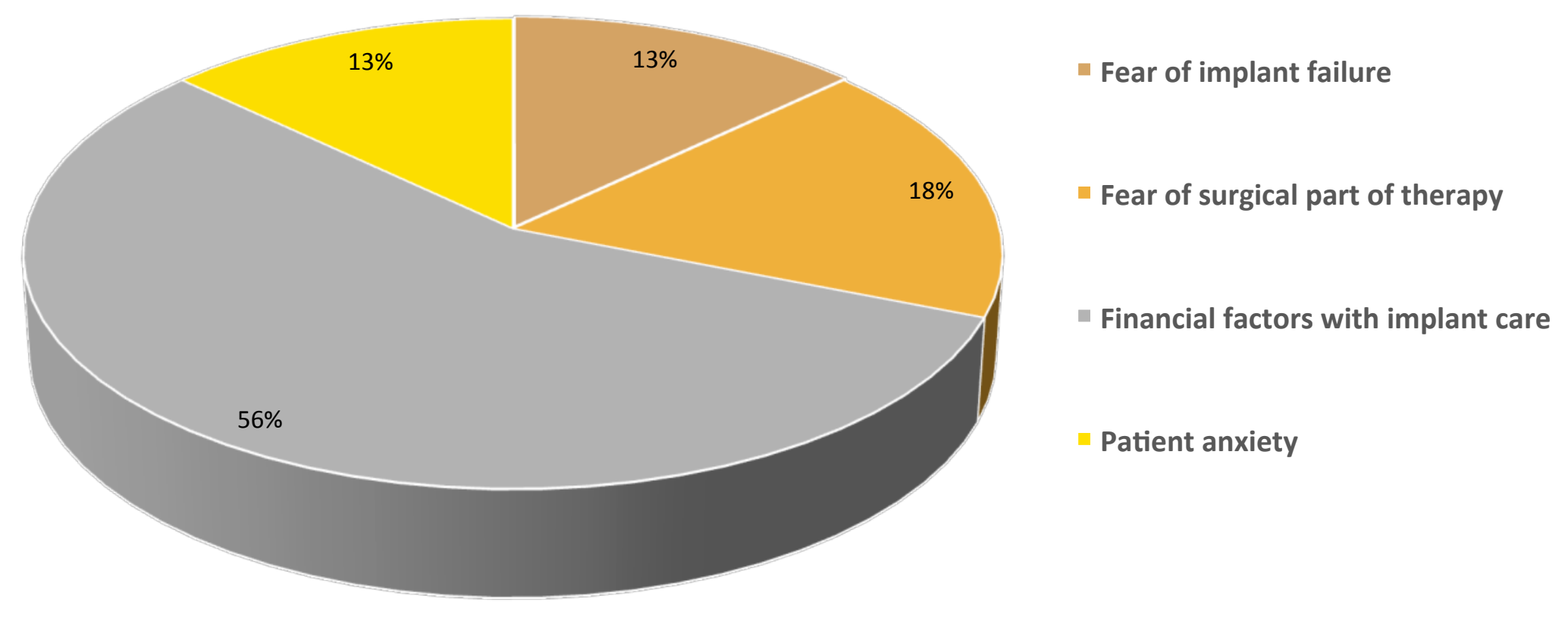

Figure 1: Social, psychological \& financial factors associated with patients decisions to have implants

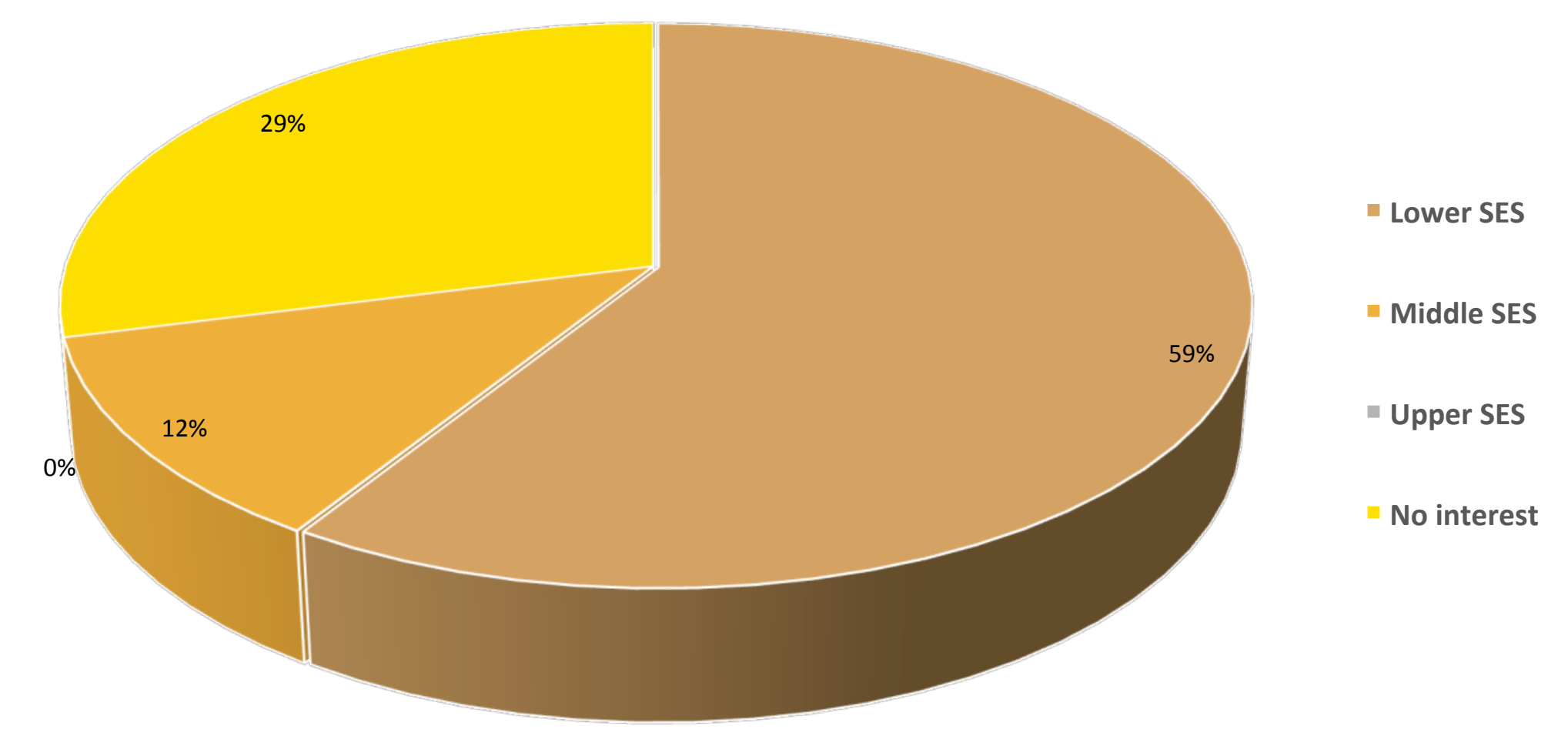

Figure 2: Patients who were interested in implants but they can't afford them

\section{Conclusions and Implications}

- The high costs of implant care influenced the patients' decisions to undergo this therapy.

- Dentists may be recommended to pay sufficient attention to the financial aspect when planning dental treatment. This might be achieved by:

(1)Emphasizing the significance of using shared decision-making in dental consultations, including providing multiple treatment options, introducing relevant treatment information and confirming patients' understanding.

(2)Developing patient's decision aid concerning implant consultation may also be highlighted.

\section{Acknowledgements}

The author acknowledge Al Baha University in Saudi Arabia for funding this study. 